\title{
El juego como alternativa para la enseñanza de conceptos básicos de salud
}

\author{
Juan Manuel Castillo Lizardo, ${ }^{1}$ Martha Rodríguez-Morán ${ }^{2}$ \\ y Fernando Guerrero-Romero ${ }^{2}$
}

RESUMEN Objetivos. Determinar la eficacia de una estrategia educativa basada en juegos populares para la enseñanza de conceptos básicos de salud en niños de edad escolar que asisten a la escuela.

Métodos. Estudio de intervención desarrollado en la ciudad de Durango, México, en junio de 2000, en el que se incluyeron 300 niños de 9 a 11 años de edad, asignados de manera aleatoria al grupo A, que utilizó una versión modificada del juego popular mexicano Serpientes y Escaleras, en la que se incluyeron mensajes sobre conceptos básicos de salud, o al grupo B,o grupo de control.

Resultados. En condiciones basales no hubo diferencias de edad, escolaridad ni sobre conceptos básicos de salud entre los niños de ambos grupos. Después de la intervención educativa la calificación sobre conceptos básicos de salud fue de $9,3 \pm 0,8$ para el grupo $A$ y de $7,5 \pm 1,1$ para el $B(\mathrm{P}<0,001)$.

Conclusiones. El uso de juegos que incluyan mensajes sobre salud e higiene puede ser una alternativa en la enseñanza de conceptos básicos de salud.

Palabras clave Educación, salud, juego, enseñanza.

Los objetivos básicos de la educación para la salud son estudiar y desarrollar procesos de transmisión de conocimientos, actitudes y valores tendientes a crear en los individuos conductas favorables a su salud (1). Las principales estrategias orientadas a la creación de mejores condiciones de salud incluyen intervenciones diri-

\footnotetext{
Unidad de Medicina Familiar No. 44, Instituto Mexicano del Seguro Social (IMSS), Delegación Durango. Durango, Dgo, México

2 Unidad de Investigación Médica en Epidemiología Clínica, Hospital Regional, IMSS. Durango, Dgo, México. La correspondencia debe enviarse a Martha Rodríguez Morán, a la siguiente dirección: Siqueiros 225 esq c/Casteñeda. Durango, Dgo, México. Tel (+52 18) 12-09-97. Fax (+52 18) 13-20-14. Dirección electrónica: rodríguez_moran@hotmail. com
}

gidas a la población infantil de edad escolar, ya que en la infancia se adquieren las actitudes y patrones de conducta que regulan la vida en la edad adulta. Teniendo en cuenta que después de los siete años los niños muestran un cambio notable en las actitudes sociales al participar en los juegos reglamentados $(2,3)$, las actividades basadas en juegos son útiles como parte de las estrategias de educación dirigidas a este sector de la población. Al respecto, en México, los libros de texto que anualmente y de forma gratuita se distribuyen a los niños de más de 6 años como apoyo para su educación básica, que abarca 9 años, contienen múltiples actividades, colectivas e individuales, en forma de juegos sencillos como apoyo didáctico en la enseñanza de las diferentes materias, incluida la educación para la salud.

El objetivo de este trabajo fue determinar la eficacia de una intervención educativa basada en el uso de un juego popular mexicano, para la enseñanza de conceptos básicos de salud en la población infantil de edad escolar que asiste a la escuela.

\section{MATERIALES Y MÉTODOS}

Para este efecto se desarrolló un estudio comparativo de intervención educativa que se llevó a cabo en junio del 2000 en la ciudad de Durango, 
Dgo., México. Tras la aceptación informada de los directivos escolares y maestros responsables, se incluyeron en el estudio 300 niños de 9 a 11 años, alumnos del $4^{\circ}$ al $6^{\circ}$ año de primaria de una escuela pública ubicada en un área urbana que atiende a una población de nivel socioeconómico medio a mediobajo. La presencia clínica de desnutrición fue un criterio de exclusión del estudio. En condiciones basales se aplicó un cuestionario estandarizado de 10 preguntas de respuesta cerrada para evaluar los conocimientos de los niños sobre conceptos básicos de salud en las áreas de salud bucal, hábitos alimentarios, hábitos higiénicos y prevención de adicciones.

Posteriormente y de manera aleatoria, los niños se integraron en uno de dos grupos: grupo A, que recibió la intervención educativa consistente en jugar una versión modificada del juego popular mexicano Serpientes y Escaleras, que consta de 100 recuadros en los se incluyeron de manera gráfica mensajes sobre salud, higiene y acciones preventivas, presentando imágenes que muestran la asociación causaefecto de acciones relacionadas con la salud. Los recuadros que contienen la "cola de la serpiente" se refieren a acciones que implican riesgo o prácticas nocivas para la salud y la "cabeza de la serpiente" representa el daño que las mismas pueden provocar. Por otro lado, "el pie de escalera" representa recomendaciones para mejorar las condiciones de salud o prevenir daños y "el final de la escalera" el beneficio que se puede alcanzar adoptando dichas acciones (figura 1). El jugador avanza las casillas según el número obtenido tras el lanzamiento de un dado. Cuando un jugador cae en una casilla que contiene el pie de la escalera se le "premia" subiendo hasta la casilla que contiene la parte superior de la escalera, y cuando cae en una casilla que contiene la cola de la serpiente es "castigado" descendiendo hasta la casilla que contiene la cabeza de la misma. En Internet puede obtenerse más información sobre este juego y sus reglas (4). Después de instruir a los niños acerca del juego, este
FIGURA 1. El juego Serpientes y Escaleras

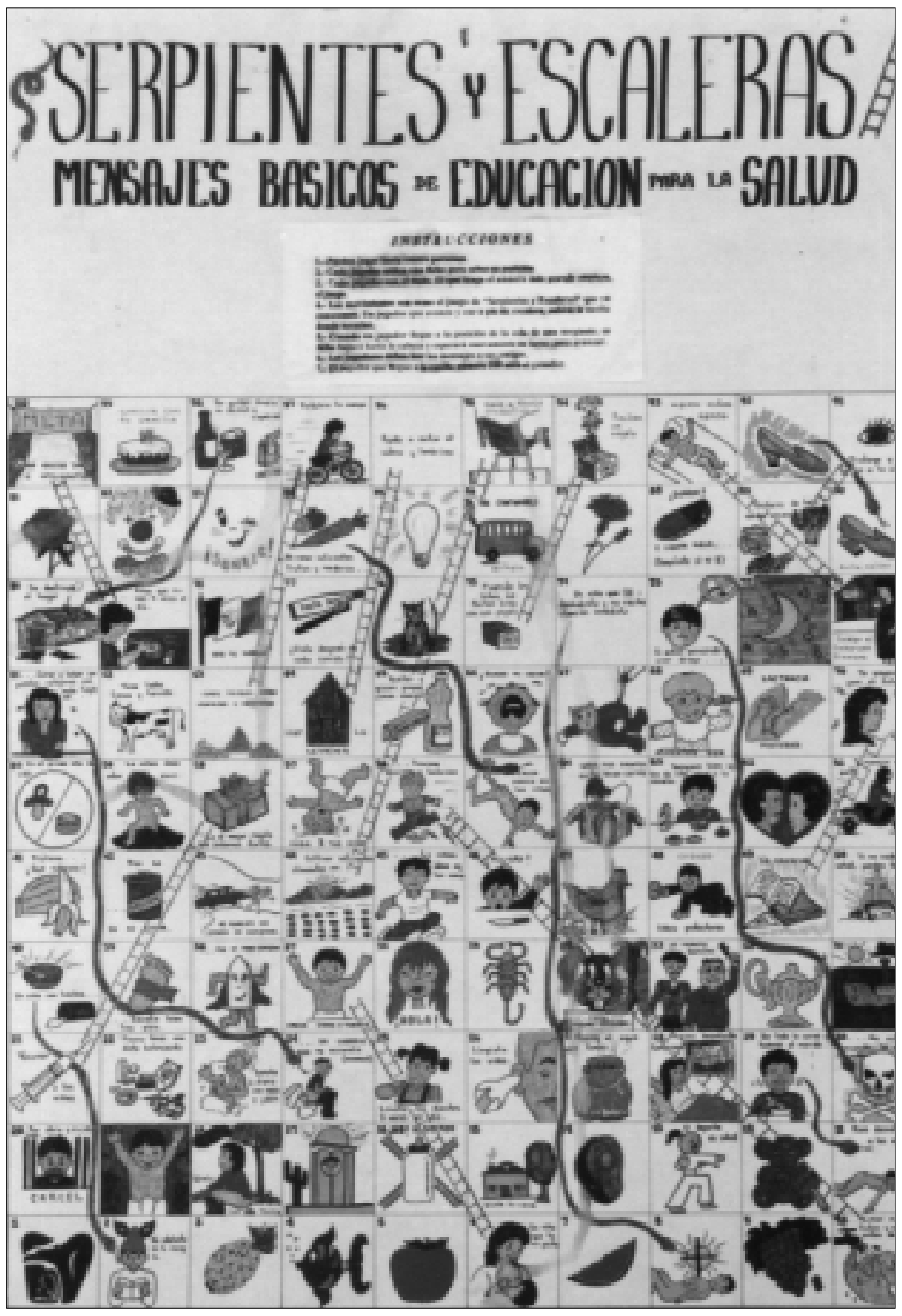

se aplicó de manera supervisada, organizando a los niños en equipos de cinco a seis integrantes por juego. Cada niño participó por lo menos en ocho sesiones de juego (máximo de un juego al día), rotando a los integrantes de los equipos en cada ocasión.

Por otro lado, el grupo B, o grupo de control, estuvo integrado por niños que recibieron en el mismo número de sesiones, pero a través de un sistema de enseñanza tradicional, la misma información sobre conceptos básicos de salud que contiene el juego de Serpientes y Escaleras utilizado. Las actividades de ambos grupos se desarrollaron en las instalaciones de la escuela durante el horario escolar (inmediatamente después del período de recreo). La instrucción y supervisión del grupo 
A estuvieron a cargo del investigador principal (JMCL), y las del grupo B a cargo de los maestros titulares de los grupos incluidos en el estudio.

Quince días después de la evaluación inicial se aplicó nuevamente el cuestionario para evaluar los conocimientos sobre conceptos básicos de salud. La falta de respuesta a una o más preguntas del cuestionario se consideró como respuesta errónea.

Las diferencias entre los grupos se estimaron con las pruebas $t$ de Student o $\chi^{2}$. Mediante el coeficiente alfa de Cronbach, se determinó la consistencia interna de los elementos del instrumento de evaluación, como estimación de la confiabilidad prueba-reprueba. Los datos fueron analizados con el programa estadístico SPSS 8.0 (SPSS Inc., Chicago Il; 1998).

\section{RESULTADOS}

Se incluyeron 150 niños en cada grupo: 80 niñas y 70 niños en el grupo A y 77 niñas y 73 niños en el $\mathrm{B}(P=$ $0,817)$. En virtud de que el estudio se desarrolló en el último mes del ciclo escolar anual, no hubo deserción de alumnos $\mathrm{y}$, por tanto, no se registraron pérdidas en el estudio.

En condiciones basales no hubo diferencias en cuanto al grado escolar (54 alumnos del $4^{\circ}, 54$ del $5^{\circ}$ y 42 del $6^{\circ}$ año en cada grupo), a la edad $(9,9 \pm 0,8$ frente a 9,8 $8 \pm 0,9$ años; $P=0,308)$ ni a la calificación media de los conocimientos sobre conceptos básicos de salud $(7,6 \pm 1,1$ frente a $7,4 \pm 1,4$ puntos, $P=$ $0,168)$ entre los niños de los grupos $\mathrm{A}$ y $B$, respectivamente.

Después de la intervención educativa, la calificación media fue de 9,3 \pm 0,8 puntos para el grupo $\mathrm{A}$, frente a $7,5 \pm 1,1$ para el grupo B $(P<0,001)$. Así, después de la intervención educativa la puntuación media aumentó en 1,7 puntos $(P<0,001)$ en los niños del grupo $\mathrm{A}$, mientras que en los del grupo B solo aumentó en 0,1 puntos $(P=$ 0,430). El instrumento de evaluación presentó un alfa de Cronbach de 0,92.

\section{DISCUSIÓN}

Los resultados de este estudio muestran que la utilización de un juego popular en el que se incluyeron mensajes sobre salud e higiene es una herramienta útil en la enseñanza de los conceptos básicos de salud en la población infantil de edad escolar. Teniendo en cuenta las características del juego Serpientes y Escaleras, su aplicación con fines educativos podría ser útil en México y otros países de América Latina que tienen problemas de salud similares.

En México, la casuística de servicios prestados en unidades de la Secretaría de Salud en 1998 (5) muestra una elevada frecuencia de consultas por problemas de salud bucal y accidentes y, a pesar de las medidas implementadas, los casos de diarrea aguda y crónica mantienen una elevada prevalencia en los niños menores de 10 años (6). Por otro lado, se ha documentado una frecuencia creciente del consumo de alcohol y drogas entre los jóvenes de 12 a 15 años de edad $(7,8)$. Lo anterior implica la necesidad de intensificar las acciones preventivas de salud dirigidas a la población susceptible, que idealmente deberían incluir estrategias para incrementar los conocimientos acerca de los conceptos básicos de salud. Al respecto, una de las técnicas para captar la atención y motivar la participación de los niños en las actividades de enseñanza se basa en la realización de juegos (3), con los que se puede lograr un mayor aprendizaje. En este estudio se utilizó una versión modificada de uno de los juegos tradicionales en la cultura popular de México, con el cual se logró incrementar de manera significativa los conocimientos sobre conceptos básicos de salud en los niños que lo jugaron.

En el proceso educativo intervienen múltiples variables socioeconómicas que pueden influir en el aprendizaje. Entre ellas cabe mencionar la nutrición y el nivel socioeconómico. Consideramos que estas variables se controlaron en este estudio al incluir niños sin signos clínicos de desnutrición que fre- cuentaban la misma escuela, a la que acuden niños que viven en la misma zona de la ciudad y con el mismo nivel socioeconómico. Sin embargo, la necesidad metodológica de controlar estas variables implica que la conclusión de que los juegos populares pueden ser útiles en las estrategias de educación para la salud solo sea aplicable a la población escolar infantil con las características señaladas. Será necesario establecer las estrategias educativas más adecuadas para los jóvenes no escolarizados ( $\sin$ hogar y sin derechos), que tienen necesidades diferentes que rebasan el objetivo de este trabajo.

El acceso a los medios de comunicación, específicamente el número de horas diarias que el niño pasa frente al televisor, es otro factor que afecta al aprovechamiento escolar. Esta variable se controló a través del proceso de asignación aleatoria a los grupos de estudio.

Otras posibles limitaciones de este estudio son la presencia no descartada del efecto Hawthorne (cambio de actitud de los sujetos en estudio cuando son blanco de interés y atención especiales) y la ya señalada con respecto a su validez externa (solo aplicable a niños que asisten a la escuela y sin problemas de desnutrición).

No medimos la persistencia a largo plazo de los conocimientos adquiridos con la intervención educativa ni su impacto sobre la modificación de actitudes y la mejoría de las condiciones de salud. Sin embargo, hay que considerar que nuestro objetivo fue solo determinar si una intervención educativa basada en juegos es útil en el aprendizaje de conceptos básicos de salud en la población infantil de edad escolar que asiste a la escuela. Es indudable que se requieren evaluaciones a largo plazo para determinar si los conocimientos adquiridos son perdurables y se reflejan en cambios de actitud y en mejores condiciones de salud, y si son necesarias acciones de refuerzo del aprendizaje, lo cual será motivo de estudios posteriores con el diseño apropiado. 


\section{REFERENCIAS}

1. Concepto y desarrollo histórico. En: Arce Gómez A, Collada Castillo R, Chávez Mayol JM, Dalhaus González AA, Heredia Duarte A, Morales Landyn L., et al. Manual de normas de educación para la salud. 2a . ed. México, D.F: Secretaría de Salud; 1976:11-13-20.

2. La infancia de siete a doce años. En: Piaget J. Seis estudios de psicología. Barcelona: Seix Barral; 1977:61-67.

3. Imitación de juegos y reglas. En: Piaget J. Seis estudios de psicología. Barcelona: Seix Barral; 1977:16-20.
4. Juegos de dados. I. Serpientes y Escaleras. http://www.hueber.de/downloads/Sprechspiel Spanish.pdf

5. Dirección General de Estadística e Informática de la Secretaría de Salud, México. Servicios otorgados en unidades de la Secretaría de Salud, 1998. Salud Publica Mex 1999;41:426-434.

6. Rodríguez-Morán M, Guerrero-Romero F. Impacto de la información masiva en la demanda de atención por diarrea en el Instituto Mexicano del Seguro Social. Cirugía 1995;4: 5-9.
7. Caraveo-Anduaga JJ, Colmenares-Bermúdez E, Saldivar-Hernández GJ. Diferencias por género en el consumo de alcohol en la ciudad de México. Salud Publica Mex 1999:41:177-188.

8. Rojas-Guiot E, Fleiz-Bautista C, Medina-Mora ME, Morón MA, Doménech-Rodríguez M. Consumo de alcohol y drogas en estudiantes de Pachuca, Hidalgo. Salud Publica Mex 1999; 41:297-308.

Manuscrito recibido el 9 de enero de 2001. Aceptado para publicación, tras revisión, el 20 de abril de 2001.

ABSTRACT Objective. To determine, for the teaching of basic health concepts to school-age children, the effectiveness of an educational strategy based on traditional children's games.

Games as an alternative for teaching basic health concepts
Methods. Intervention study carried out in the city of Durango, Mexico, in June 2000 with 300 children from 9 to 11 years old. The children were randomly divided into two groups. The children in Group A used a modified version of a Mexican popular game called Serpientes y Escaleras (Snakes and Ladders) that included messages on basic health concepts; the children in Group B made up the control group and did not play the modified game.

Results. At baseline there were no significant differences between the two groups in terms of age, grade level, or their scores on a knowledge test of basic health concepts. After the educational intervention, the health concepts test scores, out of a maximum possible of 10, were $9.3 \pm 0.8$ for Group A and $7.5 \pm 1.1$ for Group B $(P<0.001)$.

Conclusions. Using games that include health and hygiene messages can be an alternative for teaching basic health concepts. 